\title{
Tool development based on FAST for performing design optimization of offshore wind turbines: FASTLognoter
}

\author{
José E. Gutierrez ${ }^{\mathrm{a}}$, Blas Zamora ${ }^{\mathrm{b}, *}$, Julio García ${ }^{\mathrm{c}}$, María R. Peyrau ${ }^{\mathrm{c}}$ \\ a Depart. Naval Technology, Universidad Politécnica de Cartagena, Paseo Alfonso XIII 52, 30203 Cartagena, Spain \\ ${ }^{\mathrm{b}}$ Depart. Thermal and Fluids Engineering, Universidad Politécnica de Cartagena, Doctor Fleming s/n, 30202 Cartagena, Spain \\ ${ }^{\mathrm{c}}$ CompassIS, Tuset $87^{\circ} 2^{a}, 08006$ Barcelona, Spain
}

\begin{abstract}
A B S T R A C T
The new engineering FASTLognoter software is presented. This code is essentially the result of the integration of two tools, along with some additional features. The first basis tool is FAST (developed by NREL), a comprehensive aeroelastic simulator code for wind turbines. The second basis tool is Lognoter (developed by CompassIS), a general-purpose commercial software for managing and creating engi-neering forms. The integration of FAST and Lognoter gives a useful, comprehensive and versatile toolkit, which is verified in this work by means of bibliographic documented cases. An important advantage of the tool presented is the parametric design capability, allowing the user of FAST/AeroDyn/HydroDyn codes to analyze a massive group of cases; therefore, optimal design can be carried out.
\end{abstract}

\section{Introduction}

Recently, several governments, including USA, Norway, France and United Kingdom among others, have undertaken ambitious offshore wind projects. Different review works can be found on this topic (Breton and Moe [1], Wang et al. [2], Joselin Herbert et al. [3], among others). For instance, Breton and Moe [1] have discussed briefly the advantages of offshore wind energy, including a summary of the status, plans and technologies for offshore wind turbines in Europe and North America, and Wang et al. [2] have carried out a literature survey on floating wind turbines research. Some authors focused their efforts in offshore wind turbine optimization on finding the best installations and overcoming the disadvantages of this technology (Jonkman [4], Agarwal and Manuel [5], Karimirad et al. [6], among others).

\subsection{Simulation tools for onshore/offshore wind turbines}

FAST (Jonkman and Buhl [7], National Renewable Laboratory Energy, NREL) is a reference code for the study and design of wind turbines. Other codes that allow beneficial interactions with FAST have appeared, such as HydroDyn (Jonkman [4]), for the calculation of the wave loads in offshore structures, and AeroDyn (Laino and

\footnotetext{
* Corresponding author. Tel.: +34 968325 982; fax: +34 968325999. E-mail address: blas.zamora@upct.es (B. Zamora).
}

Hansen [8], Moriarty and Hansen [9]). AeroDyn, developed by Windward Engineering, is an aerodynamics software library for use in horizontal-axis wind turbine design. Besides, it is possible to find research related to the aerodynamics of wind turbines in order to validate and obtain tools that are more accurate. MEXICO and UAE experiments are two application examples of this research (Hand et al. [10], Schreck et al. [11], among others).

The most recent tools for designing wind turbines are expensive, although others, like FAST/AeroDyn, are freely available. Different commercial codes can be found, such as Bladed (GL Garrad-Hassan, www.gl-garradhassan.com), which is intended for making a complete calculation of wind turbine systems, SamCEF (Samtech, www. samtech.com), ADAMS (MSC Software Corporation, www. mscsoftware.com, NREL, www.nrel.gov, Laino and Hansen [12]) or FLEX5 (Technical University of Denmark, www.dtu.dk). Alternatively, free software for rotor aerodynamics or aeroelastic calculations of wind turbines can be found, such as QBlade (University of Berlin, www.qblade.de.to) and FAST/AeroDyn (NREL, Jonkman and Buhl [7], Laino and Hansen [8]), respectively.

\subsection{The aim of this work}

The main objective of this work is to provide a rapid prototyping software tool for studying the performance of offshore wind turbines; it means providing a new toolkit for operating with FAST/ AeroDyn/HydroDyn. This goal is achieved through the implementation of a complete form based on Lognoter (www.lognoter. 
com), a commercial software developed by CompassIS (www. compassis.com), which allows for the efficient management of information in technology. The main advantage of this tool is its capability for easily creating new Graphical User Interfaces (GUI). For this reason, Lognoter forms collection of features has been chosen to develop a new utility for integration with FAST/AeroDyn/ HydroDyn. This GUI allows the user to easily generate input files for the routines developed by NREL.

Summarizing, the new tool is called FASTLognoter, and is developed with the aim of generating and providing an interface to help the user in the normal operation with FAST/AeroDyn/Hydro$D y n$, as well as to facilitate the design optimization of wind turbines, focused mainly on offshore wind turbines.

\section{An overview of NREL codes: FAST/AeroDyn/HydroDyn}

\subsection{Background}

The FAST code simulates the non-linear dynamic performance of both onshore and offshore Horizontal-Axis Wind Turbines (HAWTs). The aerodynamic calculations of HAWTs are carried out with the AeroDyn code (Laino and Hansen [8], Moriarty and Hansen [9]). For offshore floating structures, the hydrodynamics loads are determined with HydroDyn code (Jonkman [13]), recently added to FAST. This code permits the user to model the dynamic response of conventional horizontal-axis wind turbines with both two and three blades. Additional features have been added to the FAST code

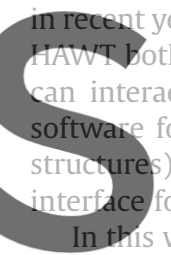
yeath
for
es), hors, which makes FAST an
ct with other codes such
or analyzing and calculating
, and other utilities such as
work, the integration of FAST
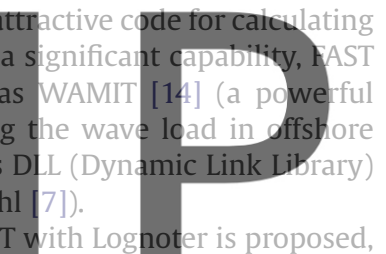
in order to achieve easier handling of the tools mentioned, obtaining FASTLognoter. Several functionalities have been added

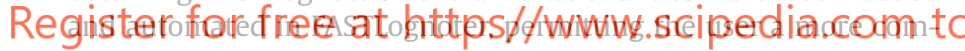
plex modeling and design optimization for wind turbines. The tool presented has new capabilities, which makes FAST/AeroDyn/ HydroDyn a more powerful simulation software.

\subsection{Brief summary of FAST/AeroDyn/HydroDyn theory}

Five relevant aspects can be highlighted in the basic theory used in the modeling with FAST/AeroDyn/HydroDyn. They are described in a summary way, and several new capabilities of FASTLognoter are described.

A) Deflection calculation. The FAST routines employ both rigid and flexible elements to model different parts of the wind turbine. For instance, the tower and the blades are considered as flexible elements. In each flexible element two mode shapes are defined, which are expressed by a sixth degree polynomial. FASTLognoter can determine the coefficients of mode shapes, through computing the outputs given by BModes, a preprocessor developed by NREL (Bir [15]), which has been added and integrated into the FASTLognoter tool, assembling in a unique software some new capacities. The structural model employed considers the flexible parts of the wind turbine as cantilever beams (Wilson [16]), with continuous mass and stiffness distributions.

B) Kinematics calculation. FAST code employs the adding angular velocity theorem to determine the angular velocity of the rotational parts of the wind turbines.
C) Kinetics calculation. The equations of motion are derived in FAST using Kane's equations [17] (which in turn are derived from Newton's Second Law).

D) Aerodynamic modeling. FAST integrates the AeroDyn routines in order to compute the wind loads on the wind turbine. When the user runs the FAST/AeroDyn codes, several parameters for computing the wind loads are required, such as aerodynamic lift, drag, and pitching moments along the wind turbine blade. AeroDyn needs data related to wind conditions, turbine geometry, and blade elements. A new preprocessor has been developed in this work, the AirFoil form (explained later), and it has been added to FASTLognoter in order to calculate the airfoil data necessary to carry out aerodynamic calculations.

The AeroDyn code allows the user to calculate the wind loads acting on the wind turbine. The code can simulate uniform or turbulent wind flow, and it uses two alternative aerodynamic models to simulate the aerodynamics of horizontal-axis wind turbines: the Blade Element Momentum theory (BEM) or the Generalized Dynamic Wake theory (GDW); in addition, it can include the static or the dynamic stall in the modeling.

E) Hydrodynamic load calculation. FAST incorporates HydroDyn for the calculation of the hydrodynamic loads acting on support platforms in offshore wind turbines. HydroDyn can simulate time-domain hydrodynamic effects from multiple parameters such as linear hydrostatic restoring, damping, non-linear vis-

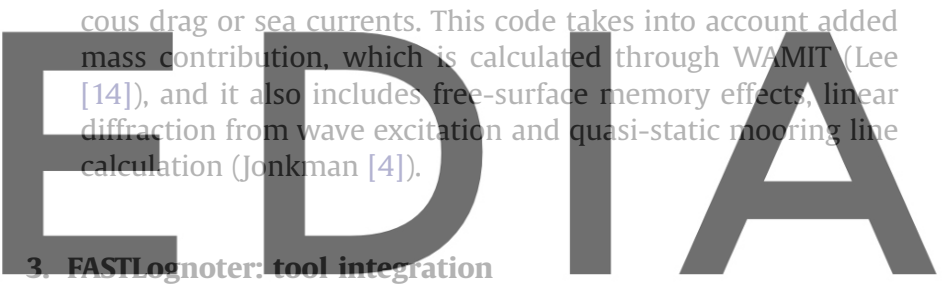

The primary idea of the tool integration is shown in Fig. 1, from and FASTLognoter. To get an operative and efficient toolkit it is necessary to take into account that to operate with FAST/AeroDyn/ HydroDyn, several pre-processors are required to achieve an accurate calculation, as is shown in this work.

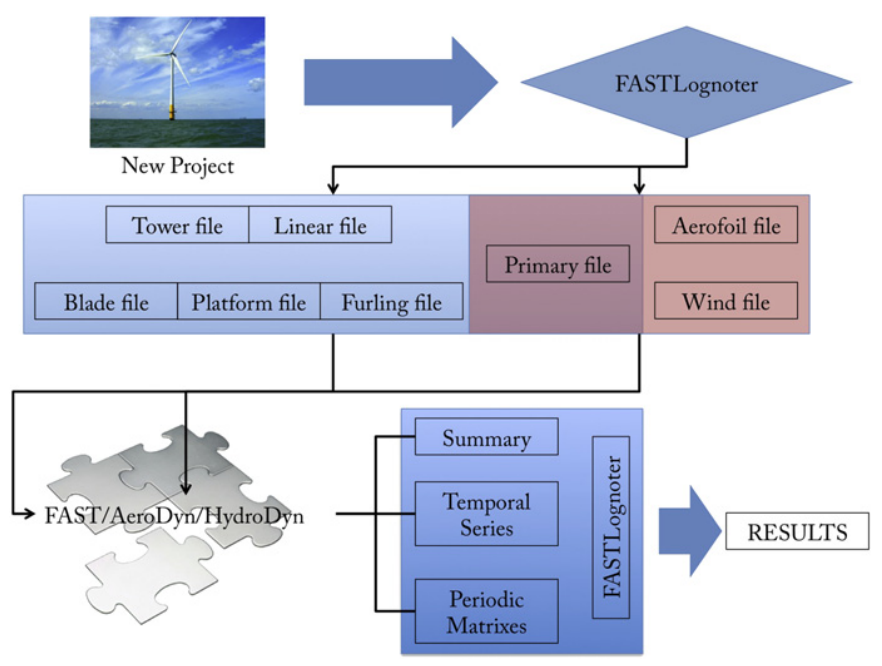

Fig. 1. Primitive idea of the tool integration proposed in this work, including the interaction between FAST and FASTLognoter. 


\subsection{An overview of Lognoter}

Lognoter can be classified as a computer tool used for the knowledge and information management of processes of different nature (in engineering or in other fields). Because Lognoter is a free programmable platform, its capabilities and resources are inexhaustible, so it can be employed for innumerable uses.

Lognoter can be used for the development and commercialization of powerful forms and user interfaces by means of the project Lognoter forms (available on the Website www.compassis.com). Furthermore, it allows the Website management, the economic management of projects and the automated creation of reports. Because the FASTLognoter tool has been developed by means of Lognoter forms, the latter capacity will be discussed later in a more detailed manner. Summarizing, Lognoter has the following important capacities: insertion of tables and pictures; database storage (SQlite) and encrustation of links; adapted sight; back-up copy; database and page encryption; design and graphics creation tools; importation/exportation; mathematical utilities; translation tools; synchronization; ease of organization and page navigation, and creation of own user interface. Advanced capacities, such as XML (Extensible Markup Language) code editing and Tcl/Tk (Tool command language/Toolkit) programming, are also included.

The code gives a clear and fast audit of the form being able to visualize operations completely. It incorporates the possibility of interactive aid; the user acquires more quickly the understanding of the interface/form. The forms of Lognoter are also placed into project Lognoter forms. This is a Website based on the Wiki concept,

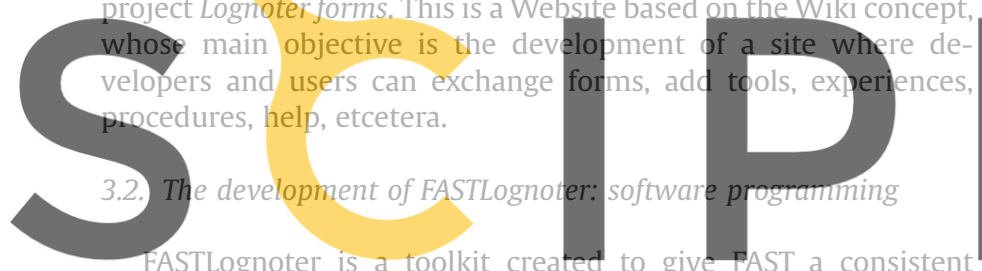
interface and to facilitate their use by beginner engineers and re-

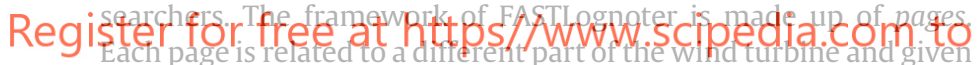
input files of FAST/AeroDyn/HydroDyn codes, which are necessary to perform the hydro-aeroelastic calculation with these codes. The new user of FAST/AeroDyn/HydroDyn codes finds it difficult to generate the files required to perform aerodynamic calculation with FAST/AeroDyn. Therefore, FASTLognoter establishes a gateway to facilitate and improve the management of these NREL codes, because it has new tools to carry out aeroelastic calculations, facilitate the file creation and the code execution, analyze the results obtained and manage and store all the information related to a new wind turbine project. The pre-processors added to FASTLognoter are related to tower structural properties (TowerProp), blade structural properties (BladeProp), calculations of the coupled shape modes (BModes), and airfoil definition (AirFoil). These tools check the values given by the users for errors. This capability facilitates the management of NREL codes, such as FAST/AeroDyn, as will be explained later.

A powerful Graphical User Interface (GUI) is developed, employing features of Lognoter like XML and Tcl/Tk programming capabilities. In addition, FASTLognoter employs an Application Programming Interface (API) of SQlite to get the input stored in the database. This toolkit gives the routines with the appropriate windows and tables in order to provide the input data for making the calculations. Furthermore, it can be difficult when using only FAST to give and obtain the appropriate parameters in order to obtain accurate computing (there are more than 600 parameters). Pre-processors are required to obtain these parameters, which model the behavior of wind turbines. For example, one needs the coupled mode shapes of the tower and blades to find the blade-tip and tower-top deflection; it is also indispensable to obtain the aerodynamic parameters of the airfoil located in the blade nodes of the wind turbine.

Lastly, FASTLognoter facilitates the creation of input files, adding into this tool-predefined cases based on NREL certificate test, which are attached together with FAST/AeroDyn codes. It also adds new features and routines to improve these codes and creates a complete toolkit for the study, design and investigation of both onshore and offshore wind turbines.

\subsection{Distinctive features of FASTLognoter}

On the one hand, FASTLognoter can classify different types of projects, organizing the information and parametric calculations of wind turbines (onshore and offshore HAWTs) depending on the input file. The page distribution of FASTLognoter allows the user to classify the parameters in the following groups: configuration, tower, blade, platform, furling, linear, aerodynamics and output parameters (see Fig. 2). To give the user more information about the software management, several bibliographic resources are included, as well as dynamic help and adapted view.

On the other hand, the projects are stored in a SQlite database and the new tool handles these projects easily (Fig. 2). This toolkit allows the user to easily add innumerable projects into a SQlite database to analyze in an individual or in a collective calculation option. Therefore, the SQlite database has a great advantage over other software, because all the projects stored can be handled

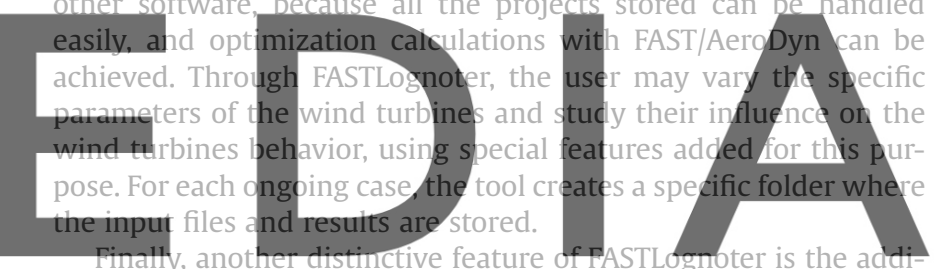

tion of predefined cases, these based on the files attached together

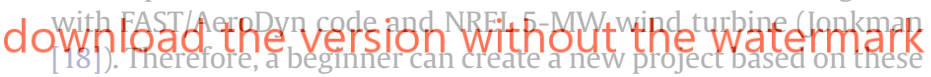
predefined cases (this capability is not included in other codes).

\subsection{Pre-processors added}

In order to help the user of NREL codes to study a wind turbine easily, several pre-processors have been added into FASTLognoter, as previously mentioned. Focusing on the tower and the blades, TowerProp and BladeProp forms are attached to FASTLognoter.

The TowerProp form allows the user to create and edit files with distributed tower properties. The files can be easily incorporated into the FASTLognoter tool, facilitating the previous calculation of the wind turbine tower. It is possible to choose several types of tower material: concrete, steel, PRGF (Polyester Reinforced with Glass Fibre) and aluminum (see Fig. 3). This form divides the tower into multiple parts and assigns the properties of each part. A linear distribution is employed to calculate the mass per unit length and other properties of the tower.

The BladeProp form makes the generation of the CSV (CommaSeparated Values) format file with distributed blade properties easier, and it can easily modify the blade properties. The files can be imported into FASTLognoter, and the blade properties can be modified by simply adding the given parameters available for that purpose (see Fig. 4). These parameters are related to flap-wise, edge-wise or torsion stiffness of the blade (Jonkman and Buhl [7]).

With regard to the modal analysis of the flexible parts of the wind turbine, FASTLognoter incorporates a pre-processor, which allows the user to obtain the coupled mode shapes of these flexible 


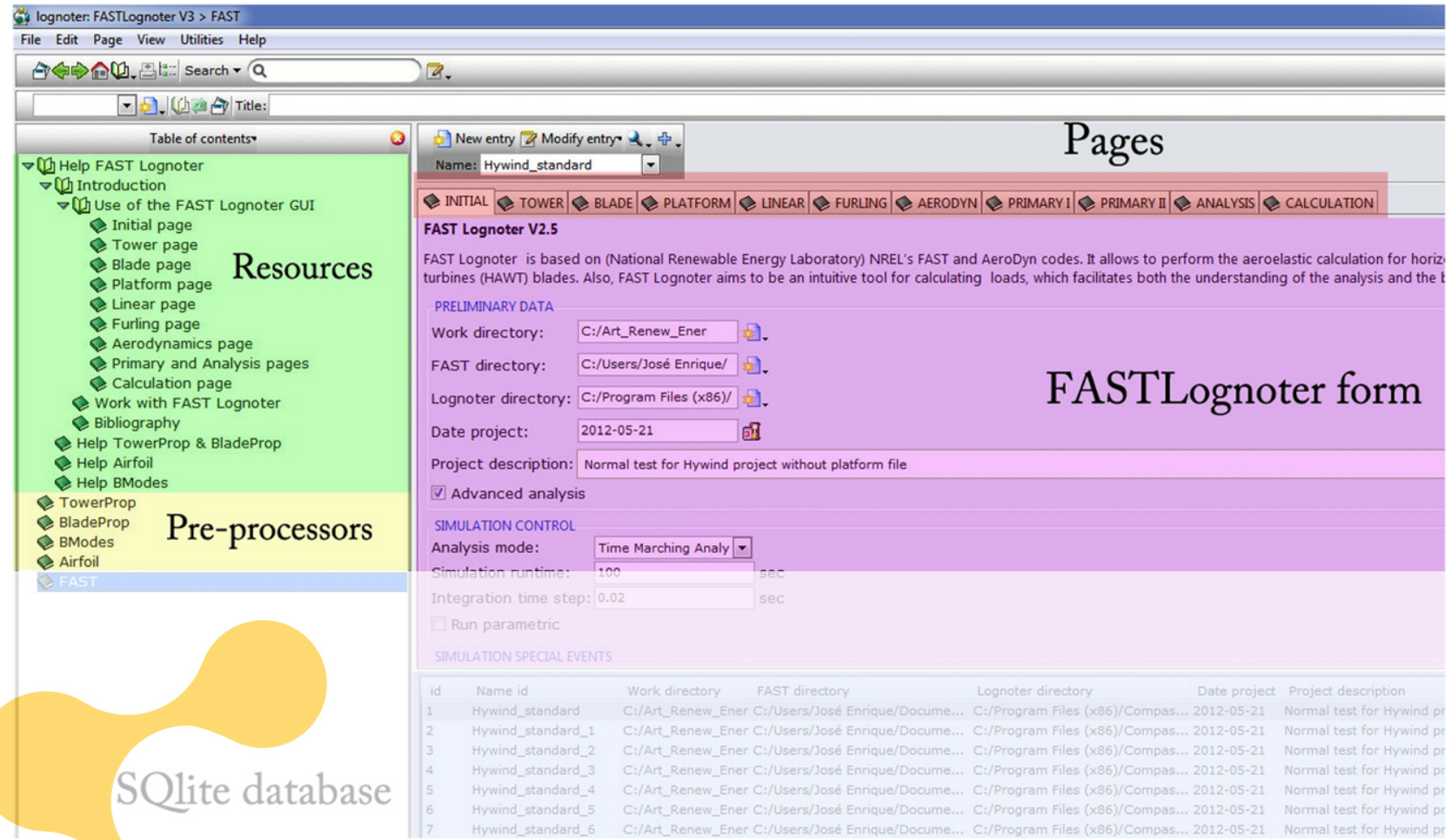

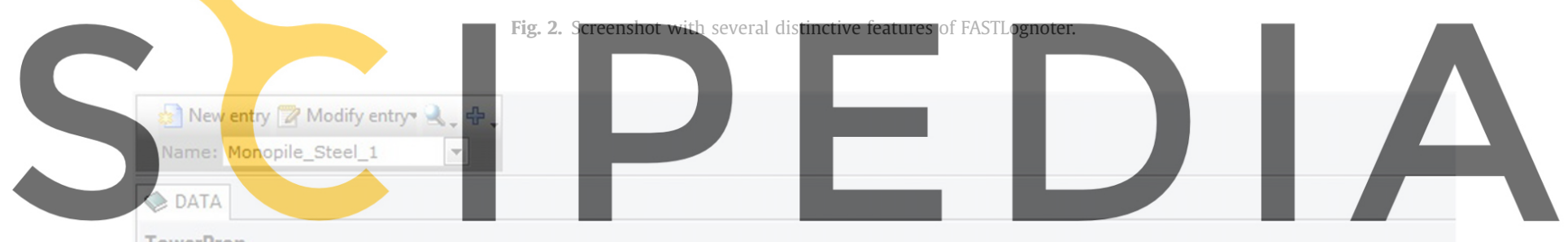

TowerProp

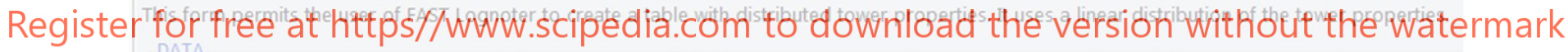
UATA

\begin{tabular}{|c|c|c|c|}
\hline Work directory: & C:llar & & \\
\hline Description: & Towe & & 4 \\
\hline Tower material: & Steel & - & \\
\hline$\square$ Vary material properti & es & & \\
\hline Input stations: & 20 & & \\
\hline Tower height: & 87.6 & & $\mathrm{~m}$ \\
\hline Base radius: & 3.25 & & $\mathrm{~m}$ \\
\hline Tickness of base tower: & 0.05 & & $\mathrm{~m}$ \\
\hline Top radius: & 1.935 & & m \\
\hline Tickness of tower top: & 0.03 & & $\mathrm{~m}$ \\
\hline
\end{tabular}

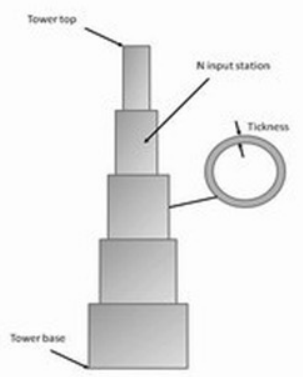

Create CSV table

$\begin{array}{llllll}\text { id } & \text { Name id } & \text { Work directory } & \text { Description } & \text { Tower material } & \text { Vary material properties } \\ 1 & \text { Monopile_Steel_1 } & \text { C:/Art_Renew_Ener/Tower_pro... } & \text { Tower made with Steel } & \text { Steel } & 0 \\ 2 & \text { Monopile_Steel_2 } & \text { C:/Art_Renew_Ener/Tower_pro... } & \text { Tower made with Steel } & \text { Steel } & 0 \\ 3 & \text { Monopile_Steel_3 } & \text { C:/Art_Renew_Ener/Tower_pro... } & \text { Tower made with Steel } & \text { Steel } & 0 \\ 4 & \text { Monopile_Steel_4 } & \text { C:/Art_Renew_Ener/Tower_pro... Tower made with Steel } & \text { Steel } & 0 \\ 5 & \text { Monopile_Steel_5 } & \text { C:/Art_Renew_Ener/Tower_pro... Tower made with Steel } & \text { Steel } & 0 \\ 6 & \text { Monopile_Steel_6 } & \text { C:/Art_Renew_Ener/Tower_pro... Tower made with Steel } & \text { Steel } & 0 \\ 7 & \text { Monopile_Steel_7 } & \text { C:/Art_Renew_Ener/Tower_pro... Tower made with Steel } & \text { Steel } & 0 \\ 8 & \text { Monopile_Steel_8 } & \text { C:/Art_Renew_Ener/Tower_pro... Tower made with Steel } & \text { Steel } & 0 \\ 9 & \text { Monopile_Steel_9 } & \text { C:/Art_Renew_Ener/Tower_pro... Tower made with Steel } & \text { Steel } & 0 \\ 10 & \text { Monopile_Concrete_1 } & \text { C:/Art_Renew_Ener/Tower_pro... Tower made with Concrete Concrete } & 0\end{array}$

Fig. 3. Pre-processors added to FASTLognoter: TowerProp (tower properties). 


\begin{tabular}{|c|c|c|}
\hline Name: Blade Hywinc & $\nabla$ & \\
\hline 8 Initial data & & \\
\hline BladeProp & & \\
\hline This form permits the & user of FASTLognoter tc & manage and create the tables with distributed blade properties \\
\hline Work directory: & C:/WorkDirectory & 8ु. \\
\hline Description: & Set here a description & \\
\hline$\checkmark$ Modify propertie & & \\
\hline Mass factor: & 1.023 & \\
\hline Thickness factor: & 1.000 & \\
\hline Flap-wise factor: & 1.000 & \\
\hline Edge-wise factor: & 1.000 & \\
\hline Torsion factor: & 1.000 & \\
\hline
\end{tabular}

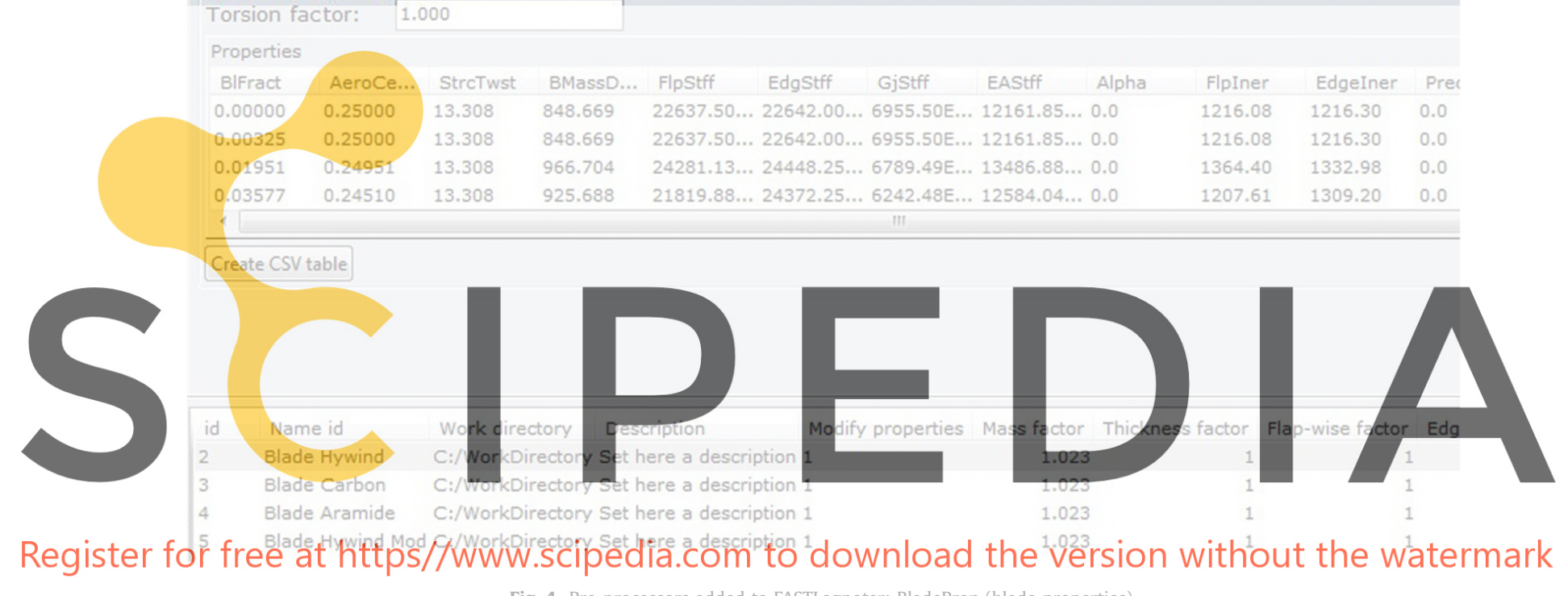

Fig. 4. Pre-processors added to FASTLognoter: BladeProp (blade properties).

parts of the wind turbine; that is to say, the tower and blades. This pre-processor is called by the same name as NREL pre-processor available for this purpose, BModes. BModes uses a finite element discretization of the flexible part of the wind turbine to carry out the modal analysis. This form obtains coupled modes for wind turbine tower and blade (see Fig. 5). A coupled mode implies the presence of coupled flexural, axial, and torsion motions in a natural mode of vibration; so, because FAST assumes a predefined mode shape approach, BModes is used. This code allows the user to set the tip-attachment. To properly set the operation with BModes, the rotor speed, blade geometry, precone, pitch control setting, tipattachment inertia properties, and distribution of structural properties the blade are needed.

Focusing on the aerodynamic parameters of airfoils, which are placed on the blades nodes, another pre-processor required by FAST/AeroDyn is AirfoilPrep (Hansen [19]). This leads to a form integrated with FASTLognoter, which allows the user to obtain the airfoil in the same way as AirfoilPrep. The pre-processor added is called AirFoil form (see Fig. 6). To obtain the aerodynamic performance of airfoils, this form uses the Viterna equations (Moriarty and Hansen [9]) for deep stall to expand aerodynamic parameters of the airfoil data for all angles from a limited set of measurements, which are required by AeroDyn. It also calculates the dynamic stall parameters needed by the Beddoes and Leishman semi-empirical model [20], which is based on airfoil indicial response. AirFoil form can interpolate the aerodynamic coefficients for other span locations, with the Du's method to augment the lift and Egger's method to modify the drag (Du and Selig [21]). In short, all the features of AirfoilPrep are included in AirFoil.

The last pre-processor added to FASTLognoter is an algorithm that makes it easier to get the coefficients of coupled mode shapes given by BModes output. These algorithms replace the excel file attached with FAST/AeroDyn codes. The coefficients of mode shapes are obtained by multiple linear regressions, usually employed to model the linear relationship between a dependent variable and one or more independent variables,

$\bar{Y} \approx \bar{X} \cdot \bar{\beta}$,

where $\bar{Y}$ is the matrix column with the displacement of the cantilever beam along undeflected tower or blade, $\bar{X}$ the matrix design and $\bar{\beta}$ the wanted matrix column with the coefficients mode shapes.

Finally, it can be stated that FASTLognoter has sizeable advantages, because it links FAST with the user, allowing the automatic creation of the input files necessary to run FAST/AeroDyn/HydroDyn codes, calculating the previous data required, classifying the files, plotting, and analyzing the results. The last advantage facilitates the interpretation of the results obtained and the study of wind turbine 


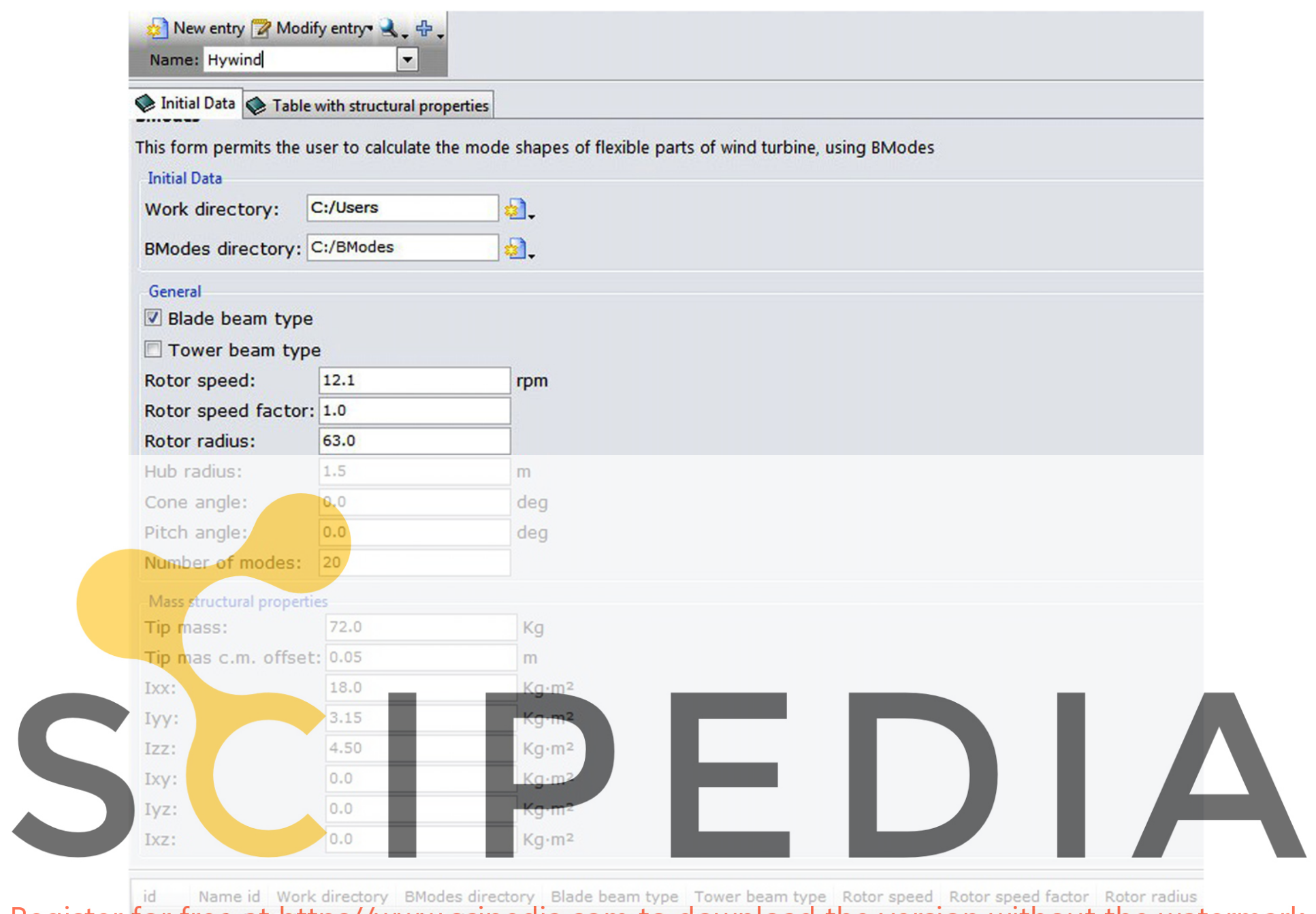

\section{Register for freenatdattps//WWW:seipedia.com to download the version without the watermark}

Fig. 5. Pre-processors added to FASTLognoter: BModes (mode shapes of flexible parts).

behavior, for both onshore and offshore types. The graphical tool added to FASTLognoter allows the user to understand and analyze the results obtained with FAST/AeroDyn codes, plotting the results into an individual or multiple adaptive graphs (see Fig. 7).

\section{Discussion of results}

\subsection{FASTLognoter verification}

It can be considered that the world's first operational deepwater wind turbine of high power (2.3 MW) is the Hywind Project Turbine (owned by the Norwegian company StatoilHydro, constructed by Siemens Wind Power), in the North Sea off Norway. In the current section, FASTLognoter is verified by comparing with other codes usually employed in the wind industry. The tests are based on the aforementioned wind turbine, with an offshore spar buoy wind turbine of power 5-MW.

Fig. 8 shows a representative comparison between the results obtained by using several codes and interfaces, following the IEA Wind Annex XXIII-Subtask 2, a benchmark test under the coordination of NREL. In addition to the results obtained in this work for the tower-top fore-aft shear force with FASTLognoter, Fig. 8 shows the results obtained by NREL, CENER (National Renewable Energies
Center, Spain), CWMT (Fraunhofer Center for Wind Energy and Maritime Engineering, Germany), Risø DTU (National Laboratory for Sustainable Energy, Denmark) and Siemens AG (Germany), with different codes: Bladed (based on modal analysis), ADCoS (based on finite element method), BHAWC (based on non-linear finite element method) ADAMS, HAWC and FAST (based on multibody dynamics formulation). The results are achieved with $6.9 \mathrm{rpm}$ rotor velocity and $8 \mathrm{~m} / \mathrm{s}$ steady inflow wind velocity. The GDW model for computing the loads on the turbine is employed, as well as the Beddoes-Leishman dynamic stall model; in addition, the aerodynamic pitching moments of the blades and the velocity deficit in the wake of the tower have been taken into account. It can be seen that the results obtained in this work follow a similar trend to those reported by NREL; the slight discrepancy may be due to differences in the input data.

\subsection{Application case: a design investigation of blades based on NREL 5-MW offshore wind turbine}

This application case, a design optimization study, is proposed to find better blade behavior in a given offshore wind turbine. In order to achieve this aim, NREL 5-MW offshore wind turbine (Jonkman et al. [22]) is proposed as a basis for the design 


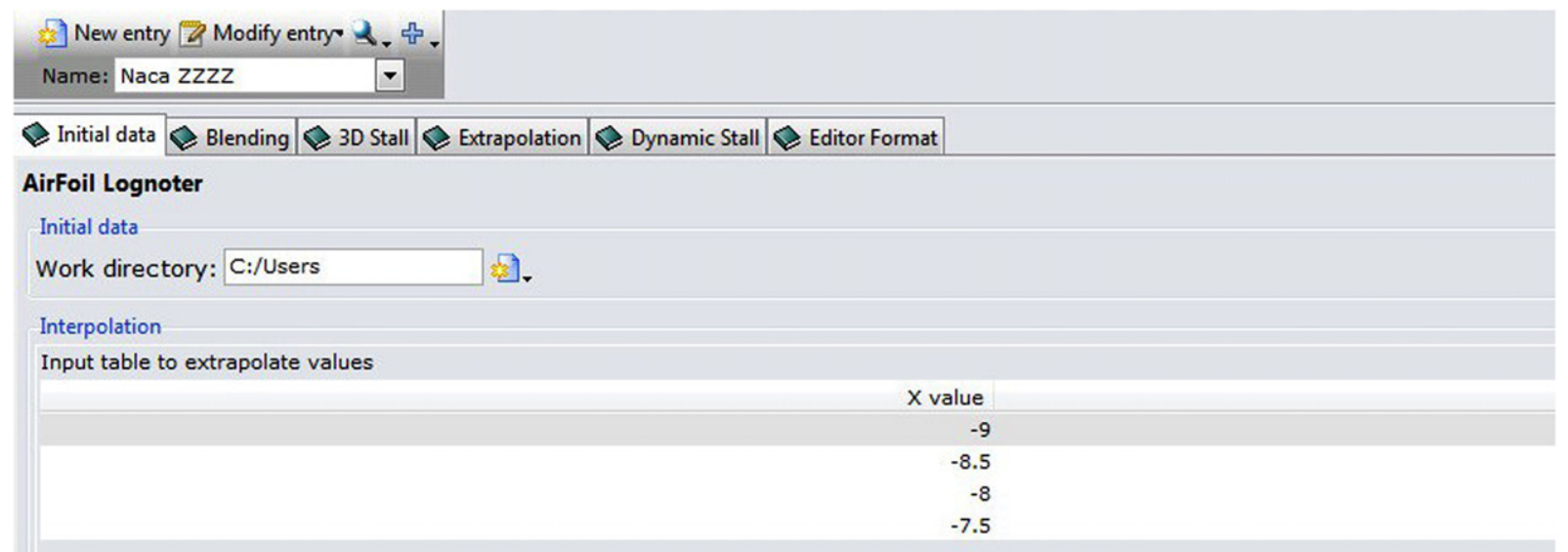

Initial stage: -25

Final stage: 25

Increment:

Interpolate
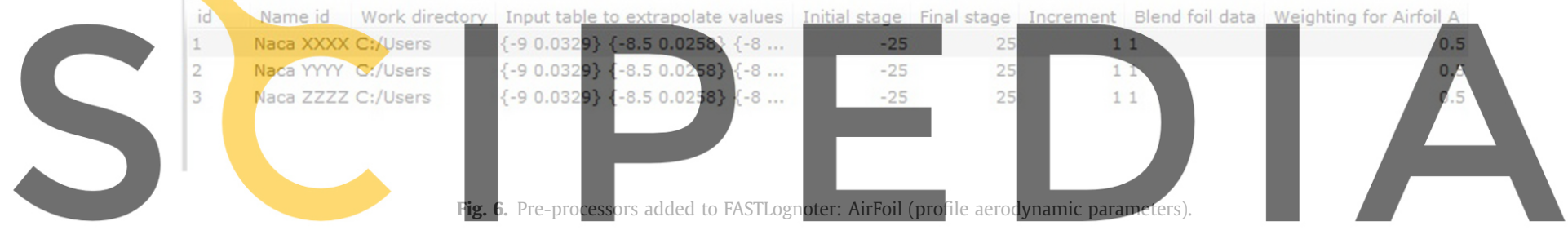

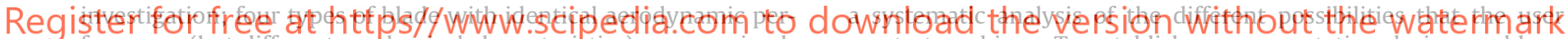
formance (but different mechanical characteristics) are examined.

The design optimization process with FASTLognoter is described schematically in Fig. 9. Although final design decisions should be stated taking into account additional considerations such as cost, visual impact or noise reduction, this new tool can perform

\section{wants to achieve different types of blade are considered:}

- Blade Type 1. This is the basis blade, based on the NREL 5-MW Baseline wind turbine, made of PRGF.

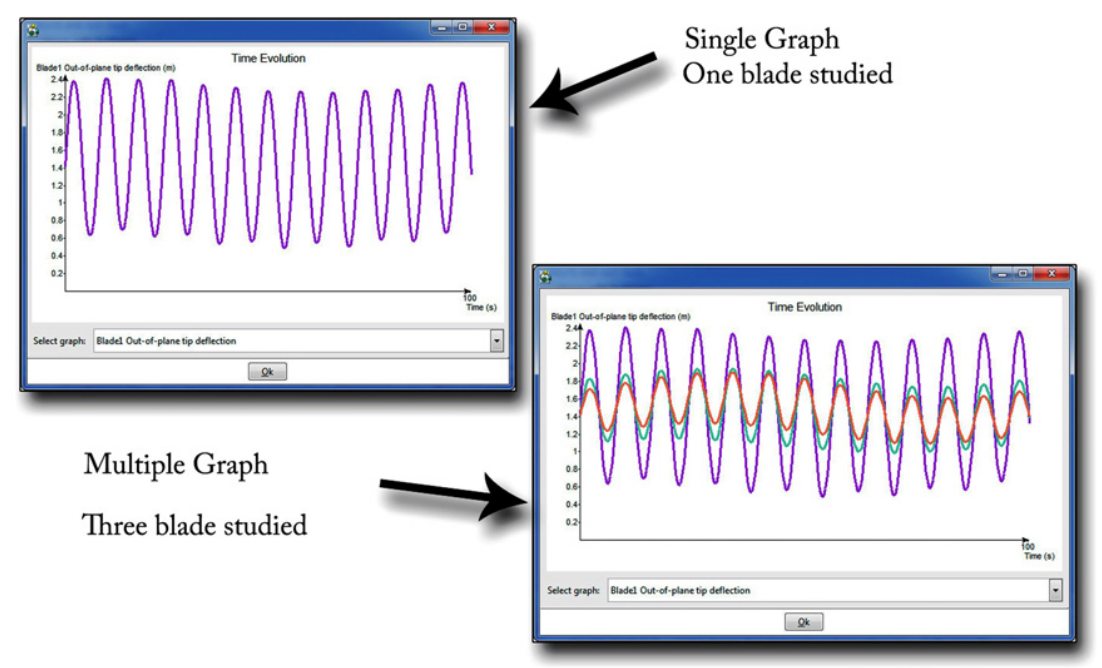

Fig. 7. Typical graphs obtained in FASTLognoter 


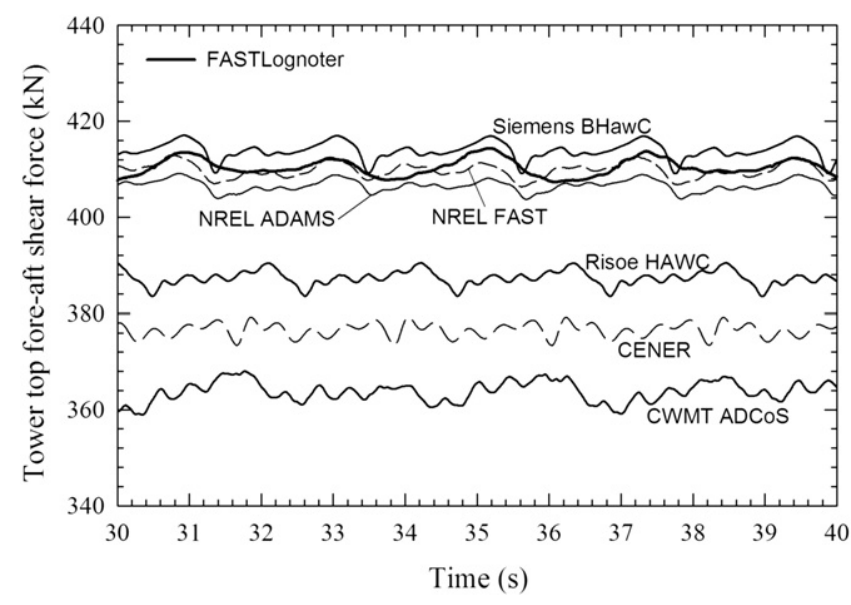

Fig. 8. Comparison between the results with FASTLognoter and those obtained with different companies and researchers, using several codes (IEA Wind Annex XXIIISubtask 2, OC3-Hywind Turbine 5-MW).

- Blade Type 2. As Blade 1, but made of carbon fibre.

- Blade Type 3. As Blade 1, but made of aramide.

- Blade Type 4. As Blade 1, but with $25 \%$ increased thickness.

The blade mass and stiffness distribution of Blades $1-4$ are shown in Fig. 10a-c. As expected, Blade Type 4 is the heaviest blade,

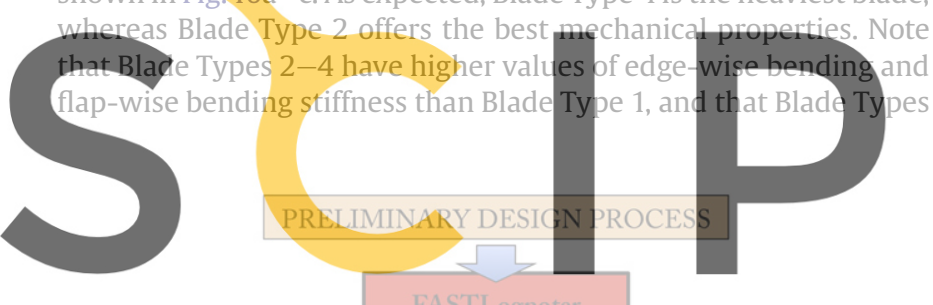

Register for free athittps h/WWWWAscipedia.com

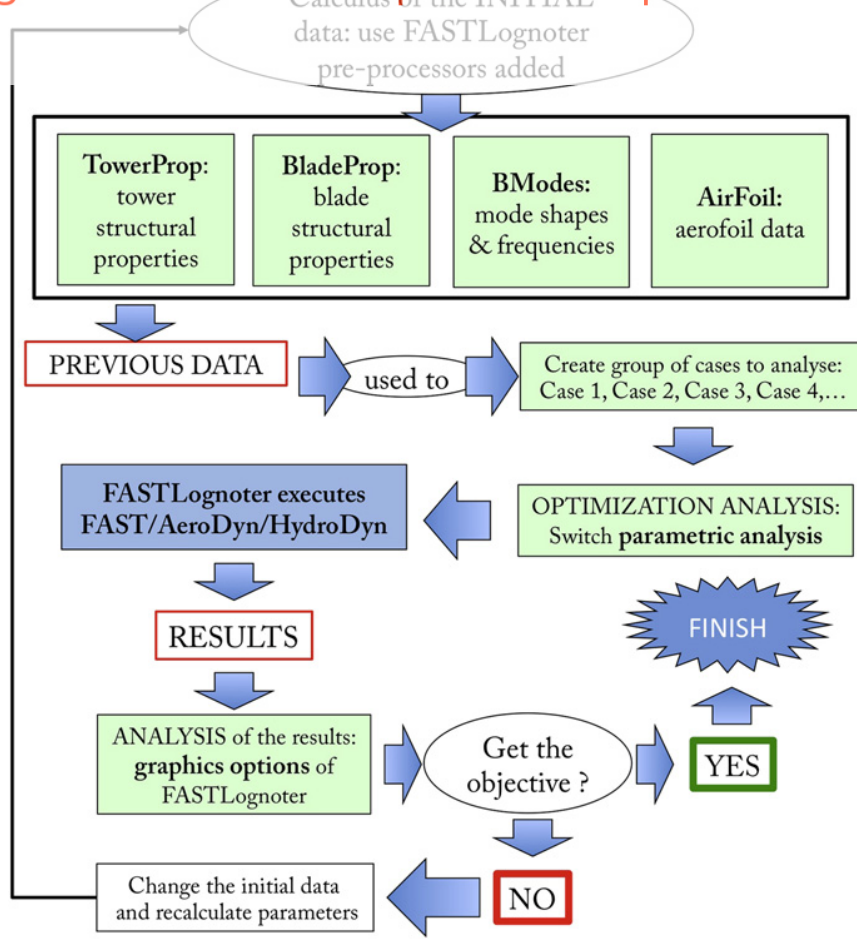

Fig. 9. Scheme of the design optimization process with FASTLognoter.
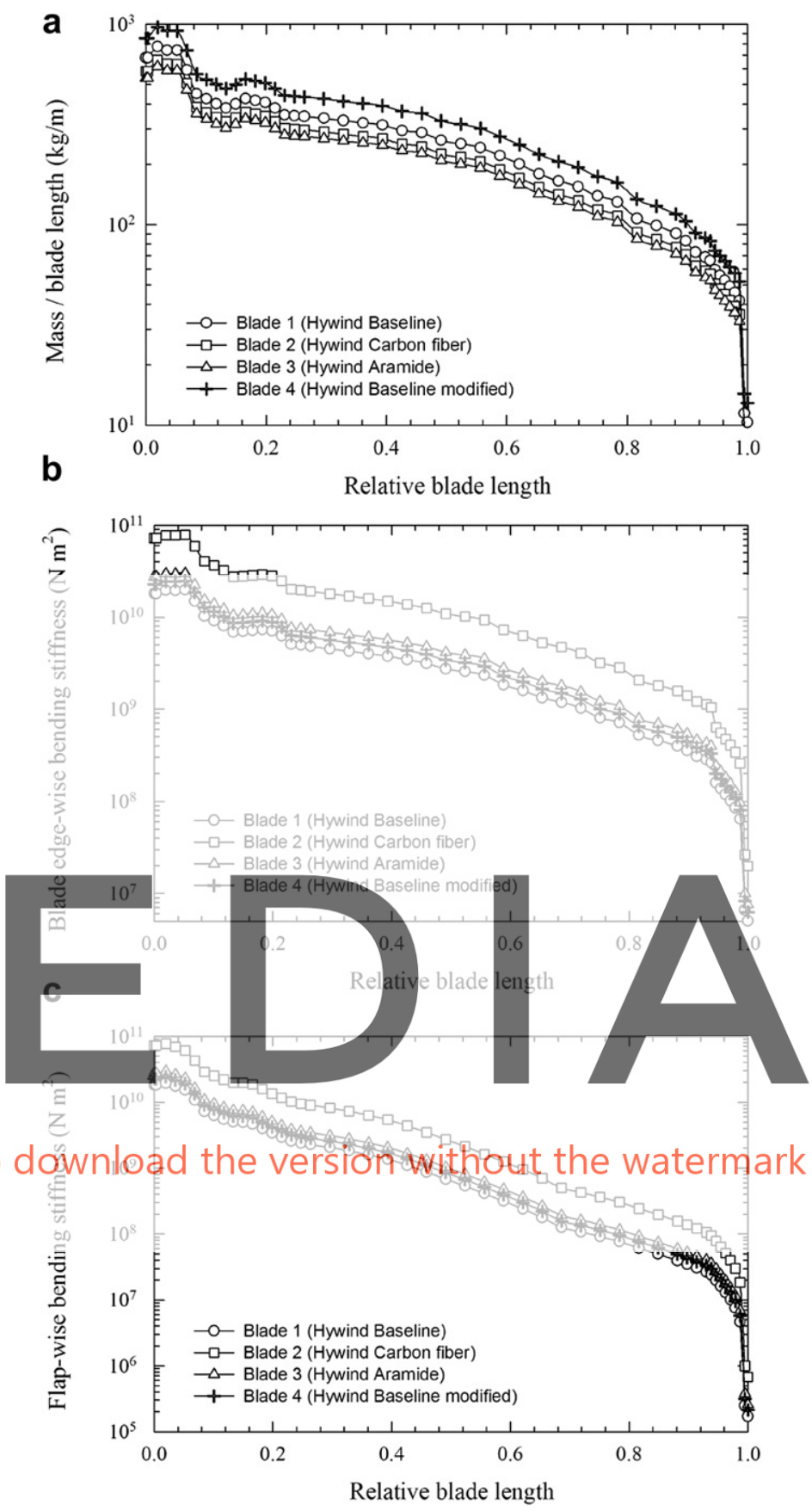

Fig. 10. Mass and stiffness properties of the proposed Blade Types 1-4. a) Mass per unit of blade length. b) Edge-wise bending stiffness. c) Flap-wise bending stiffness.

2 and 3 achieve a mass reduction compared to the reference Blade 1.

The behavior of the blades has been studied under the same axial wind flow, with values of $1-20 \mathrm{~m} / \mathrm{s}$. Several relevant parameters calculated through FASTLognoter are presented: blade edgewise tip deflection, blade flap-wise tip deflection, tip to tower clearance and axial tip deflection. Each blade is divided into 17 nodes and the mechanical characteristics are presented per unit length. To carry out the proposed investigation, it is necessary to get data using pre-processors added into FASTLognoter toolkit (TowerProp, BladeProp, BModes and AirFoil). 


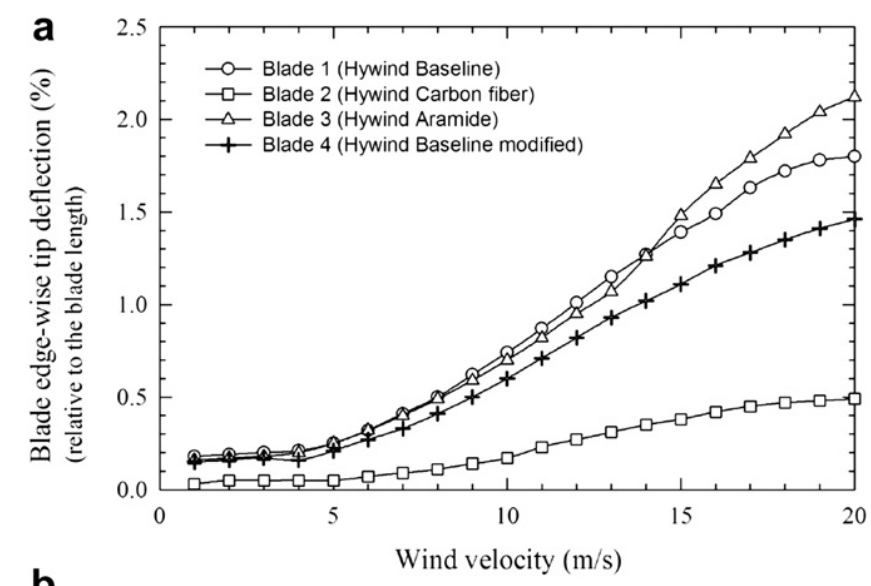

b

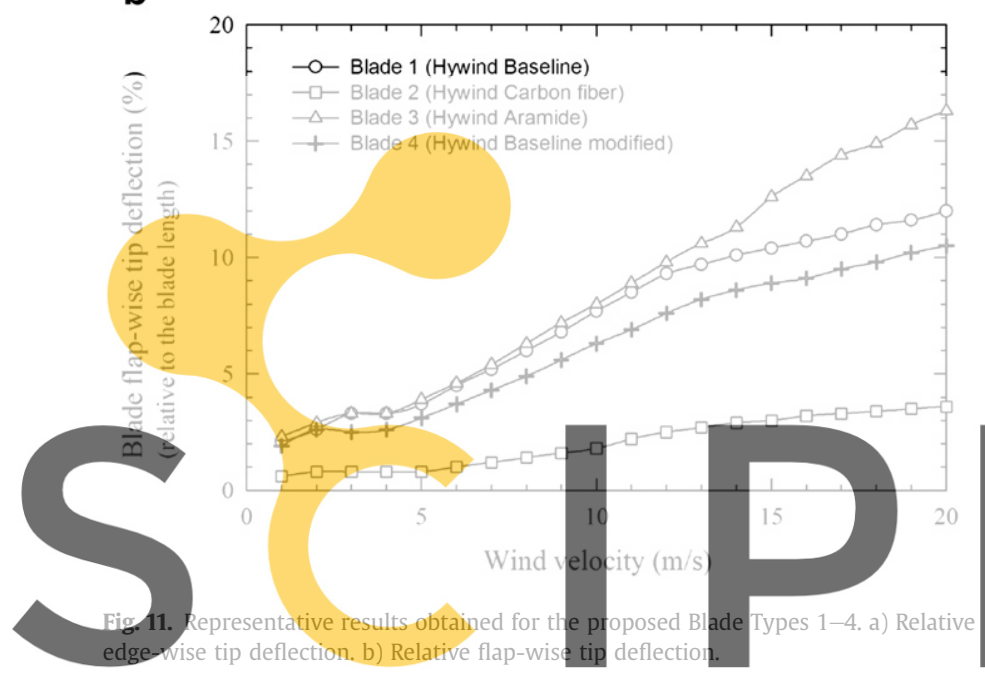

a

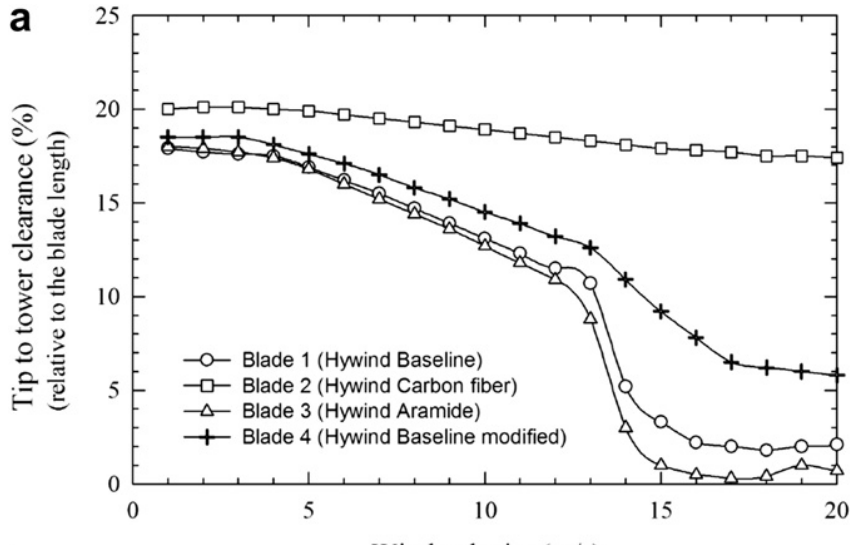

b

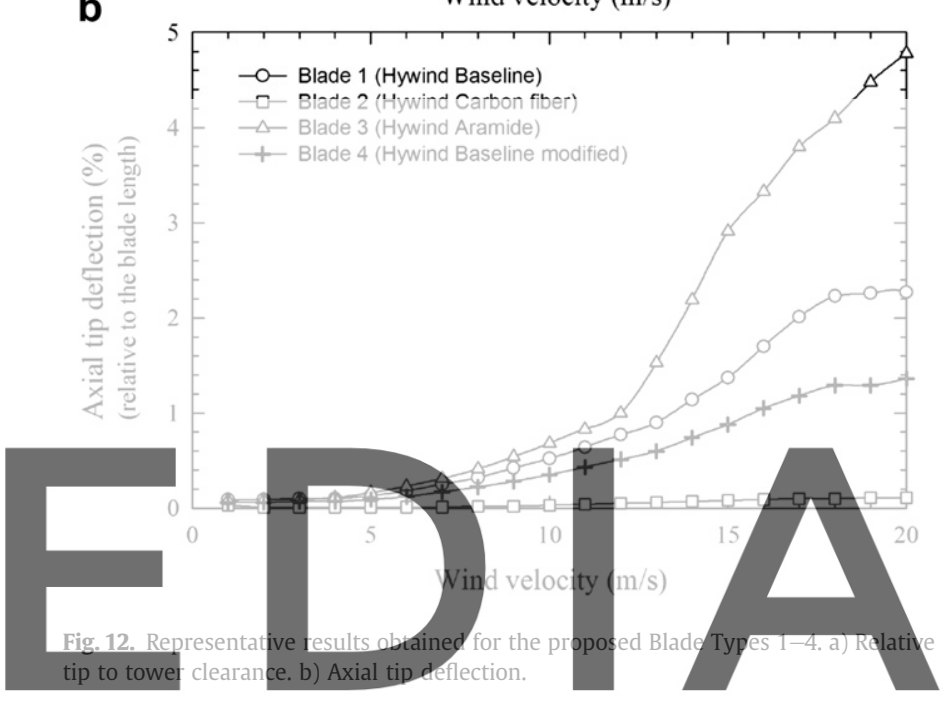

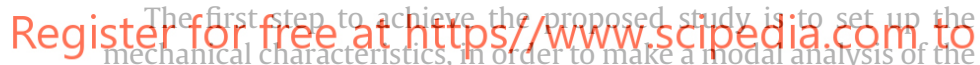

coupled mode shapes. This allows the user to obtain the first and second flap-wise bending mode and first edge-wise bending mode of each blade analyzed. For this purpose, BModes is used. Then, these data are used in FASTLognoter to create a blade file appropriate to run FAST/AeroDyn. For each case analyzed, it is necessary to establish a new entry in SQlite database of FASTLognoter, and then a new case is stored. When all the cases are prepared, the option parametric analysis is selected to carry out the complete calculation of all the cases given to FASTLognoter. With respect to the timing, a simulation time has been established following the rules given by the International Electrotechnical Commission procedure (61400-1, Wind turbine generator systems) on the normal operation of a wind turbine. A time increment given as $\mathrm{d} t=\mathrm{d} \Theta / \omega$ (being $\Theta$ the azimuth step and $\omega$ the rotor speed) has been assigned. Thus, the results presented belong to the last minute of the simulation in each case.

The results obtained can be observed in Figs. 11 and 12. The evolution of blade deflection versus wind velocity can be seen. The influence of the stiffness on the blades behavior is clear. To a great extent, the increase of the parameters related to type of material or airfoil thickness reduces the tip deflections. The relationship between the mass and the best mechanical behavior is optimized in carbon fibre. Indeed, Blade Type 2 has better performance than the original blade based on NREL 5-MW wind turbine (Blade Type 1), achieving the desired goal, i.e., to improve the tip deflection of wind turbine blade along with a relevant mass reduction. In this case, the carbon fibre blade obtains a sizeable $23.5 \%$ in mass reduction.

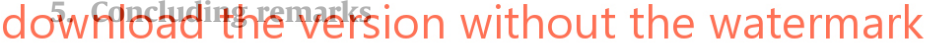

- The present work gives response and solution to an existing need in wind energy engineering; the tool presented, FASTLognoter, provides a graphical user interface for FAST, for carrying out a complete study on wind turbines (both onshore and offshore).

- This software helps the beginner engineer and the inexperienced developer to work in the field of renewable energy.

- Several pre-processors have been added to make FASTLognoter a competitive toolkit, permitting the user to calculate the required data and carry out a complete analysis of a wind turbine: TowerProp, BladeProp, BModes and AirFoil.

- A key feature of the new toolkit presented is the capability to achieve parameterized studies and design optimization.

- It can be concluded that several useful features of FAST have been significantly enhanced through FASTLognoter.

\section{References}

[1] Breton SP, Moe H. Status, plans and technologies for offshore wind turbines in Europe and North America. Renew Energy 2009;34:646-54.

[2] Wang CM, Utsunomiya T, Wee SC, Choo YS. Research on floating wind turbines: a literature survey. IES J Part A Civil Struct Eng 2010;3(4):267-77.

[3] Joselin Herbert GM, Iniyan S, Sreevalsan E, Rajapandian S. A review of wind energy technologies. Renew Sustain Energy Rev 2007;11:1117-45.

[4] Jonkman JM. Dynamics modeling and loads analysis of an offshore floating wind turbine. Technical Report NREL/TP-500-41958. Colorado, USA: National Renewable Energy Laboratory, www.nrel.gov; 2007.

[5] Agarwal P, Manuel L. Simulation of offshore wind turbine response for longterm extreme load prediction. Eng Struct 2009;31(10):2236-46. 
[6] Karimirad M, Meissonnier Q Gao Z, Moan T. Hydroelastic code-to-code comparison for a tension leg spar-type floating wind turbine. Marine Struct 2011:24:412-35.

[7] Jonkman JM, Buhl Jr ML. FAST user's guide. Technical Report NREL/EL-50038230. Colorado, USA: National Renewable Energy Laboratory, www.nrel.gov 2005.

[8] Laino DJ, Hansen AC. Users guide to the wind turbine aerodynamics computer software AeroDyn. Spanish Fork, USA: Windward Engineering LC, www. windwardengineering.com; 2002.

[9] Moriarty PJ, Hansen AC. AeroDyn theory manual. Technical Report NREL/TP500-36881. Colorado, USA: National Renewable Energy Laboratory, www.nrel. gov; 2005.

[10] Hand MM, Simms DA, Fingersh LJ, Jager DW, Cotrell JR, Schreck S, et al. Unsteady aerodynamics experiment phase VI: wind tunnel test configurations and available data campaigns. Technical Report NREL/TP-500-29955. Colorado, USA: National Renewable Energy Laboratory, www.nrel.gov; 2001.

[11] Schreck S, Sant T, Micallef D. Rotational augmentation disparities in the MEXICO and UAE phase VI experiments. Conference Paper NREL/CP-50047759. Colorado, USA: National Renewable Energy Laboratory, www.nrel.gov; 2010.

[12] Laino DJ, Hansen AC. User's guide to the computer software routines AeroDyn interface for ADAMS. Colorado, USA: Winward Engineering, National Renewable Energy Laboratory, www.nrel.gov; 2001.

[13] Jonkman JM. Modeling of the UAE wind turbine for refinement of FAST-AD. Technical Report NREL/TP-500-34755. Colorado, USA: National Renewable Energy Laboratory, www.nrel.gov; 2003.
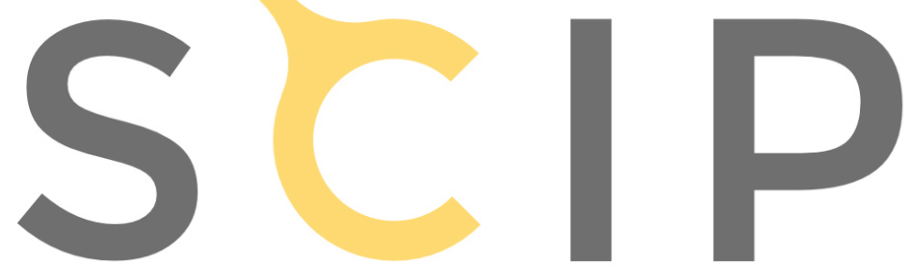

[14] Lee CH. WAMIT theory manual. Report No. 95-2. Cambridge (Mass.), USA Department of Ocean Engineering, Massachussets Institute of Technology; 1995

[15] Bir GS. User's guide to BModes (software for computing rotating beam coupled modes). Colorado, USA: National Renewable Energy Laboratory, www. nrel.gov; 2007, http://wind.nrel.gov/designcodes/preprocessors/precomp/ PreComp.pdf; 2007 [accessed 16.07.2012].

[16] Wilson RE, Walker SN, Heh P. Technical and user's manual for the FAST-AD advanced dynamics code. NREL Report 99-01. USA: Oregon State University: 1999.

[17] Kane RT, Levinson DA. Dynamics: theory and applications. New York: McGraw-Hill; 1985.

[18] Jonkman JM. Definition of the floating system for phase IV of OC3. Technical Report NREL/TP-500-47535. Colorado, USA: National Renewable Energy Laboratory, www.nrel.gov; 2010.

[19] Hansen AC. NWTC design codes (AirfoilPrep). http://wind.nrel.gov/ designcodes/preprocessors/airfoilprep/. [accessed 16.07.2012].

[20] Leishman JG, Beddoes TS. A semi-empirical model for dynamic stall. J Am Helicopter Soc 1989;34:3-17.

[21] Du Z, Selig MS. A 3-D stall-delay model for horizontal axis wind turbine performance prediction. In: ASME wind energy symposium, and 36th AIAA1998-21 aerospace sciences meeting and exhibit, Reno (Nevada), USA 1998. p. $12-5$.

[22] Jonkman JM, Butterfield S, Musial W, Scott G. Definition of a 5-MW reference wind turbine for offshore system development. Technical Report NREL/TP500-38060. Colorado, USA: National Renewable Energy Laboratory, www nrel.gov; 2009 